\title{
A Selecting Robots Method USING VISIBLE LIGHT COMMUNICATION
}

\author{
Wataru Uemura, Kenta Shimizu \\ Department of Electronics and Informatics, Ryukoku University, Shiga, Japan
}

\begin{abstract}
From the view of Industry 4.0, robots and machines like as automated guided vehicles and manufacturing robots are focused on in order to achieve factory automations which changings from mass production to variant variable production. Managing them are important task for workers. For this environment, remote control is required to manage these devices, however, it is often difficult to know the identification number of them. Therefore, we propose a selecting system where we can select the controlled target device by using visible light communication. The transmitter sends its own identification number by the visible light communication and transmits the control command using wireless communication like as Wi-Fi. At this time, the control command is transmitted by the broadcast communication to all the devices connected to the same network. The device selected by the visible light communication receives the identification number of its transmitter, and the device can execute the control command on Wi-Fi which has the same identification number in its packet. In order to evaluate the complexity of device selection, we have some experiments which measures the operation time to select devices with unknown identification number. From this experimental result, the proposed method is considered to be the easy-to-use remote controller with the least operation error.
\end{abstract}

\section{KEYWORDS}

Visible Light Communication, Remote Control, Wireless Communication

\section{INTRODUCTION}

Automated guided vehicles and manufacturing robots are focused on in order to achieve factory automations which changings from mass production to variant variable production [1-2]. The main task of workers at factory will changing from assembling products to managing robots and machines that works such assembly of products. For this environment, wireless communication is very important because remote control is needed in order to manage these devices such as robots and machines (shown as Figure 1). However, since the locations of these devices are changed due to the factory line recombination, it is often difficult to know the identification number of them. Therefore, we propose a novel selecting system where we can select the control target device by using visible light communication. By visible light communication for selecting the target to be controlled, we can select the devices that are inside the illuminated range by the transmitter, so it is possible for us to select the target easily and intuitively.

In this paper, we propose the novel selecting system using visible light communication. In Section 2 , we introduce the modulation method of visible light communication. And Section 3 describes the proposed system to select targets. The performance of the proposed selecting method is shown in Section 4 and we conclude this paper in Section 5. 


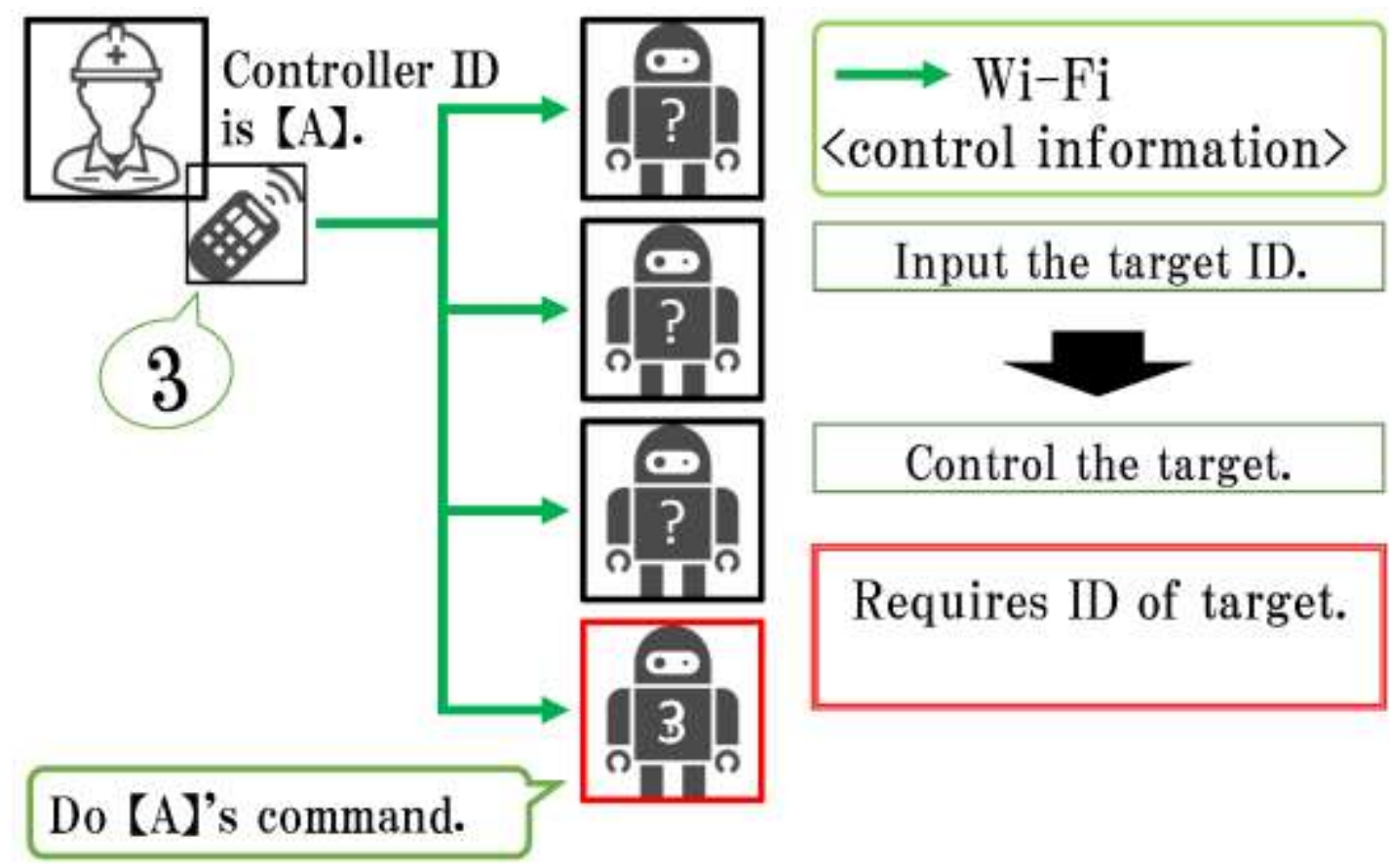

Figure 1. A control method using remote controller in order to control multiple devices.

\section{Visible Light COMMUNICATION}

Visible light communication is one of wireless communication methods and uses visible light in order to send information [3-5]. And visible light communication is suitable for the selflocalization system because it has very strong directivity [6]. This communication method is not handled by the radio law, so the robot controlling system using visible light communication in the hospital are proposed [7]. For the communication by visible light, changing the blinking pattern of the visible light according to the sending information. Figure 2 shows information allocation by inversed 4-Pulse Position Modulation method (i-4PPM). The $\mathrm{x}$-axis means the time and one slot is very short time like as $10 \mathrm{~ms}$. And the y-axis means the voltage of the light such as LED. So, the yellow area means the bright by LED.

Since the human eyes seems that always turning on because of its high speed, so we can use it as a lighting device like a flashlight. Because the receivable area is equal to the illuminated area by visible light communication, the user can see the wireless communication area. And visible light communication has the feature of strong directivity, it is not found in other wireless communication methods [8-10].

As these reasons, this communication method is suitable for the selection of the targets.

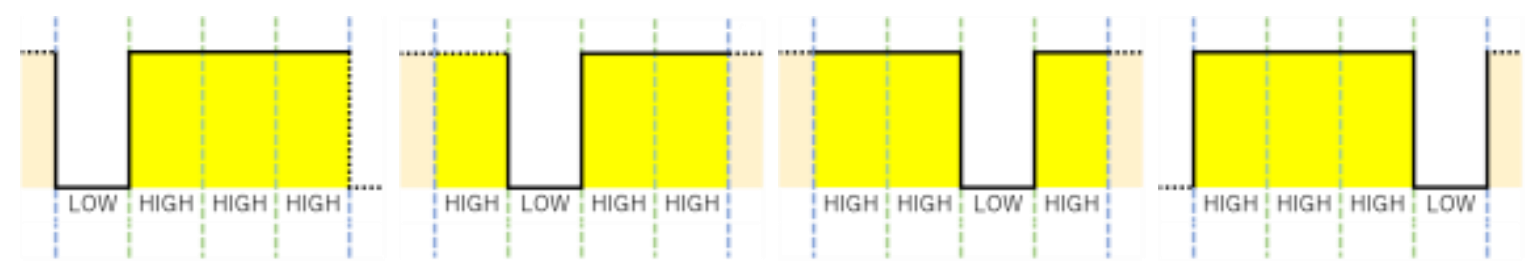

Figure 2. Using four blinking patterns can send four different information like as 2 bits. 


\section{Proposed Method to Select the Controlled Target by Visible Light COMMUNiCATION}

In this section, we propose the control method which uses the remote controller using visible light communication to select the illuminated device by the light. A block diagram of the proposed system is shown in Figure 3. The operator sends its controller's ID to robots by the visible light communication (shown as red dotted line). And at the same time, the operator sends its command to all robots via Wi-Fi (shown as green line). The only robots which received the ID can do the command. That means the operator can control the lighted up robots.

The transmitter sends its own identification number by the visible light communication and transmits the control command using wireless communication like as Wi-Fi. At this time, the control command is transmitted by the broadcast communication to all the devices connected to the same network. Figure 4 shows the transmitter circuit of our system.

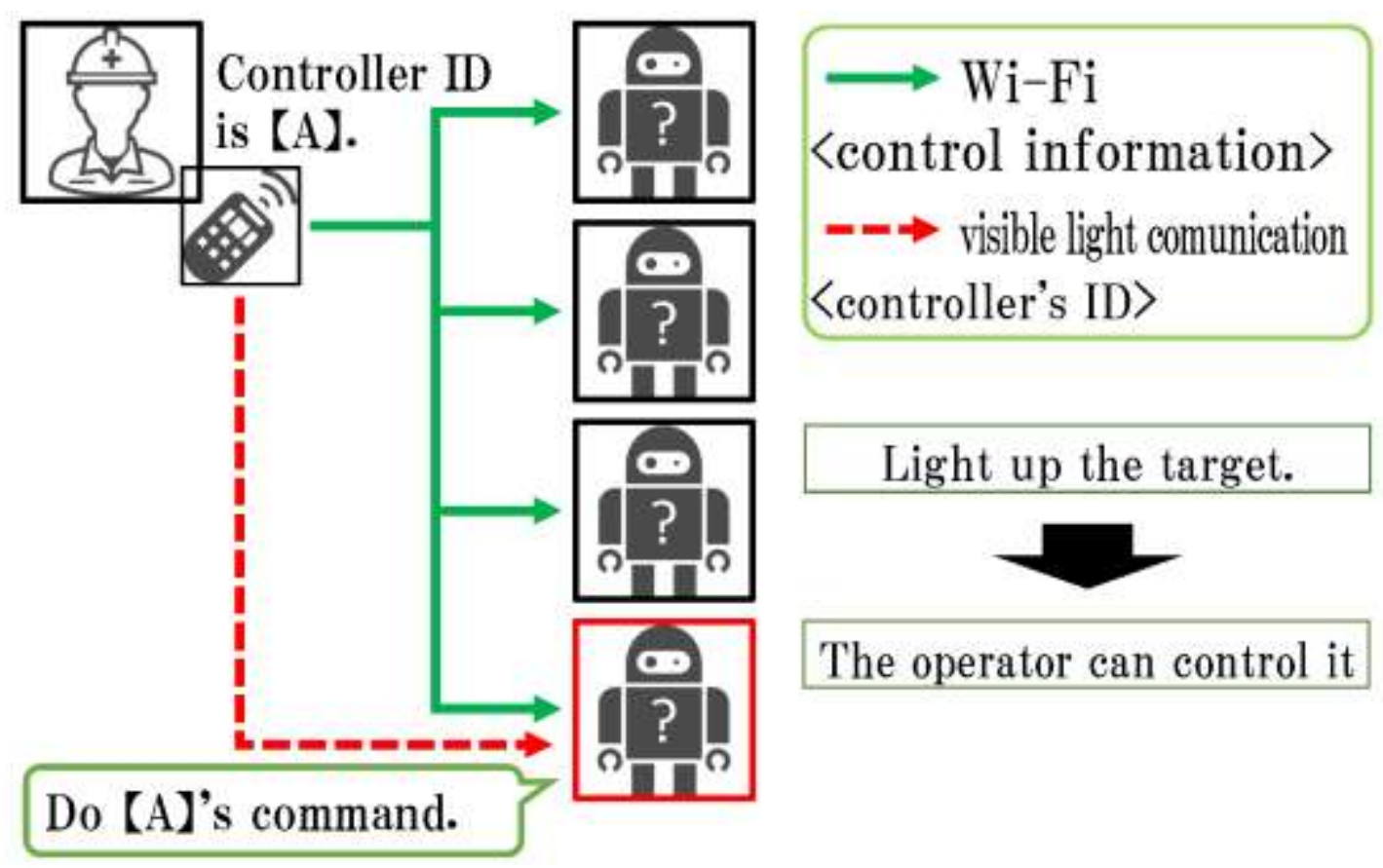

Figure 3. Outline of proposed robot control system using visible light communication (shown as the red dotted line).

The device selected by the visible light communication receives the identification number of its transmitter, and the device can execute the control command on Wi-Fi which has the same identification number in its packet. Figure 5 shows the receiver circuit of our system.

Figure 6 shows our prototype system to select and control the remote devices using visible light communication. The control information sent by the controller is always transmitted to all the devices existing in the same network by broadcast communication via the access point from Raspberry Pi. By pushing the button on the controller, the microcomputer like as the arduino transmits its identification number using visible light communication with LED. When the photodiode on the receiver gets its information by visible light communication, the 
International Journal of Ad hoc, sensor \& Ubiquitous Computing (IJASUC), Vol.9, No.3/4, August 2018 microcomputer compares its number and own number. If they are same number, the controller accepts its command.

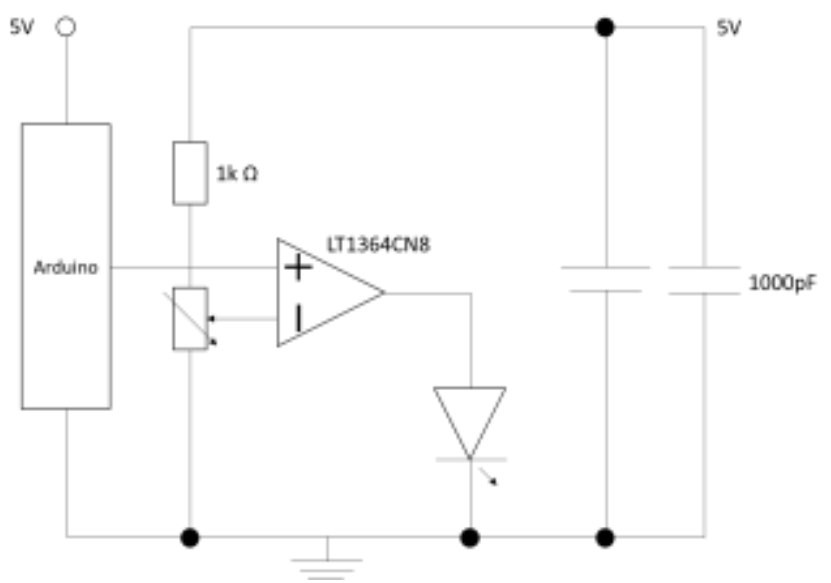

Figure 4. The transmitter circuit of our prototype system using visible light communication [11].

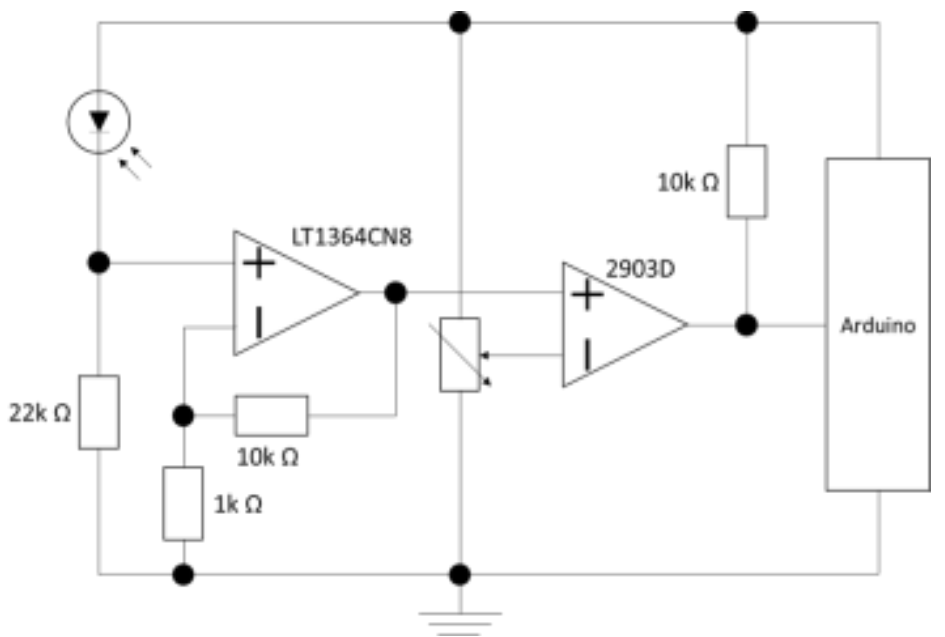

Figure 5. The receiver circuit of our prototype system using visible light communication. 


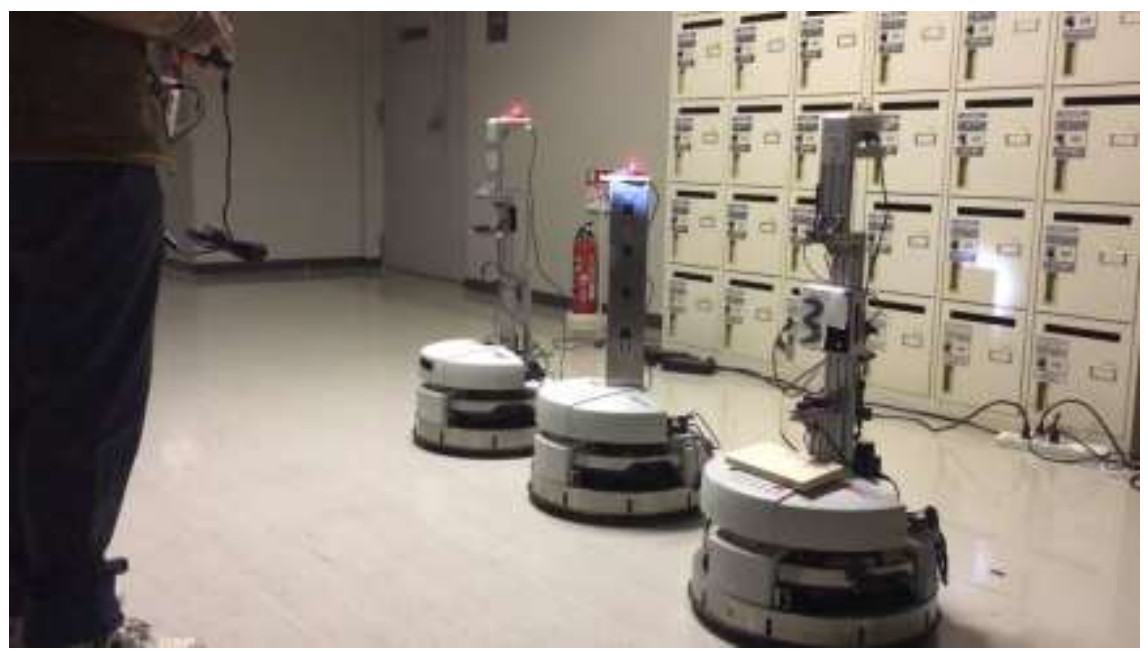

Figure 6. The overview of our prototype system. The operator has the controller and the transmitter for visible light communication. And he selects the target robot by illuminating it.
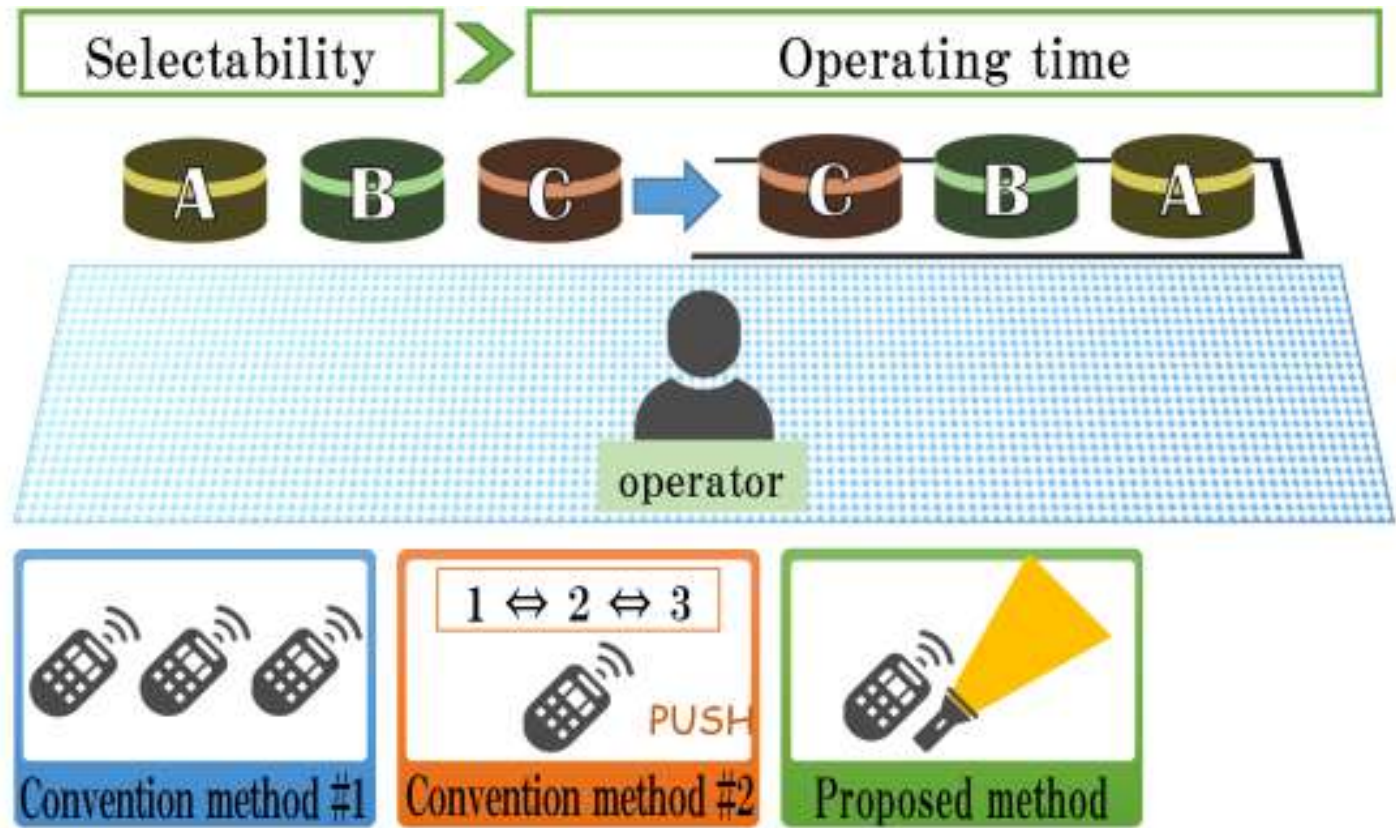

Figure 7. The experimental environment.

We compare the operating time for changing the order of robots.

\section{EXPERIMENTAL RESUltS}

In order to evaluate the complexity of device selection, we have some experiments which measures the operation time to select devices with unknown identification number. Three omnidirectional mobile robots are used for this experiment.

At first, we prepare a field as shown in Figure 7-left. And we place three omnidirectional mobile robots in it, and call it 1st, 2nd and 3rd from the left, respectively. Second, the operator moves these three omni-directional mobile robots to arrange them so that they locate $3 \mathrm{rd}, 2 \mathrm{nd}$, and $1 \mathrm{st}$ from the left, respectively as shown in the Figure 7-right. We have the rule that does not allow for 
the robots to move across the bold line, thereby the operator must select target robots at least five times.

We measure the operating time using following three kind of selection method for four operators.

\section{- Conventional method 1}

A method to select controllers corresponding to each of the three robots. These controllers look like the same, so the operator cannot know which controller can communicate with which robot.

\section{- Conventional method 2}

A method to select controlled robot by buttons. Operators cannot know which button corresponds to which robot.

\section{- Proposed method}

A method to select controlled robot by visible light communication. Operator need not known robots' identification numbers.

Figure 8 shows the experimental results. Conventional method 1 took a lot of time because it is necessary to change the remote controllers. Moreover, with the three robots, there was no big difference in average time between the conventional method 2 and the proposed method.

We should need the number of robot become more over. However, the proposed method has the smallest range of operating time. From this fact, the proposed method is considered to be the easy-to-use remote controller with the least operation error.

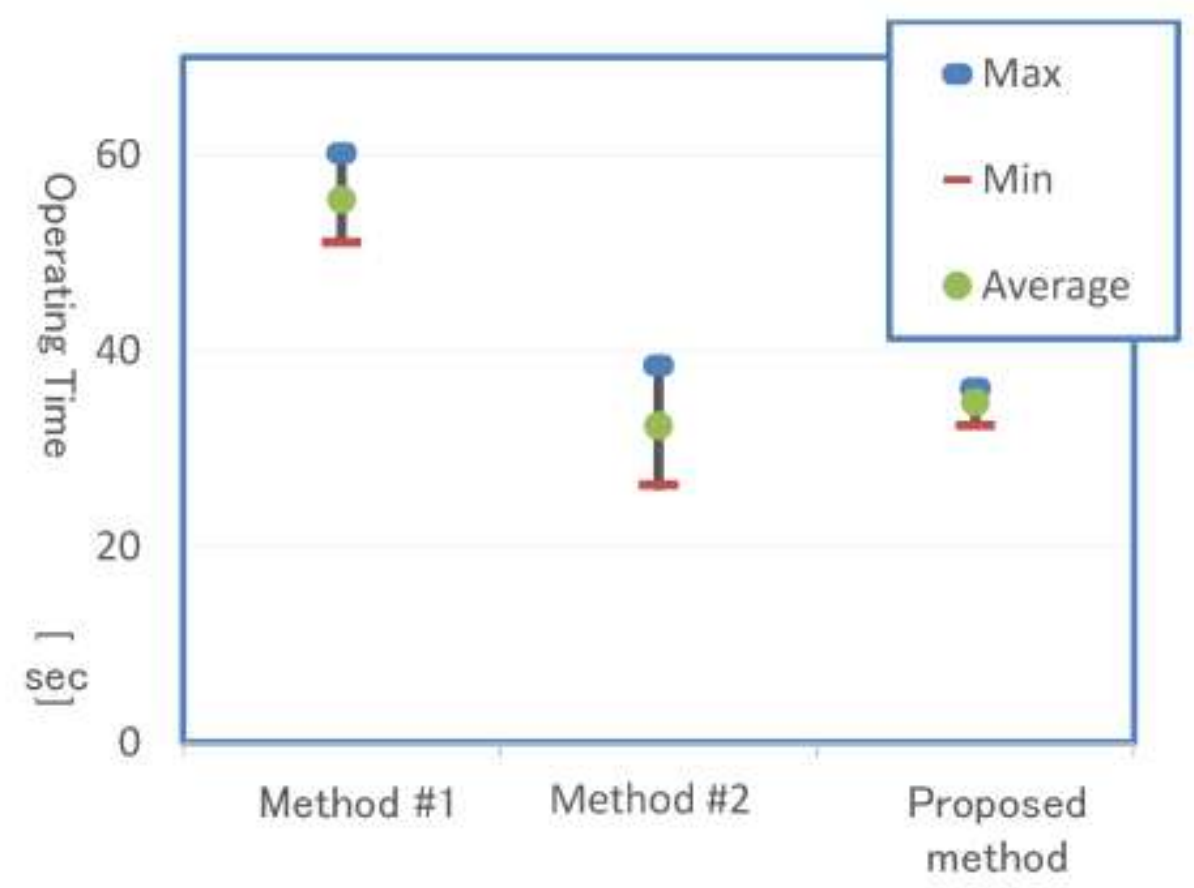

Figure 8. The experimental results.

This figure shows the comparison of operating time on exchanging robot placement. 


\section{Conclusion}

For the factory automation, robots and machines like as automated guided vehicles and manufacturing robots are focused. Managing them are important task for workers. In this paper, we propose a selecting method to control them using visible light communication. Since it can be chosen visually by the proposed method, it is an easy-to-use controller that does not cause selection errors even if the number of devices increases. In addition, although the proposed controller enables simultaneous control of multiple devices, in the simultaneous movement of a plurality of devices, unless the directions of the devices are in agreement, there is a problem that the moving directions do not coincide with each other. Therefore, this is our future work.

\section{REFERENCES}

[1] Tim Niemueller, et al., "The Carologistics Approach to Cope with the Increased Complexity and New Challenges of the RoboCup Logistics League 2015," Automation, Communication and Cybernetics in Science and Engineering 2015/2016, pp. 619-635, 2015.

[2] World Robot Summit - Assembly Category: http://worldrobotsummit.org/en/programs/ challenge/?id=industrial, 2018/07/07 accessed.

[3] S. Haruyama,"Visible light communication," Journal of IEICE (D), vol. 94, no. 12, pp. 1055 - 1059 , 2011.

[4] Rajan Sagotra, "Visible Light Communication," International Journal of Computer Trends and Technology (IJCTT) vol. 4, No. 4, pp. 906 - 910, 2013.

[5] Dominic C. O'Brien, "Visible Light Communications: challenges and possibilities," PIMRC 2008. IEEE 19th International Symposium on Personal, Indoor and Mobile Radio Communications, pp.15 $18,2008$.

[6] H. Sharifi, A. Kumar, F. Alam and K. M. Arif, "Indoor localization of mobile robot with visible light communication," 2016 12th IEEE/ASME International Conference on Mechatronic and Embedded Systems and Applications (MESA), Auckland, 2016, pp. $1-6$.

[7] R. Murai, T. Sakai, H.Kawano, Y. Matsukawa, Y. Kitano, Y. Honda, and K. C. Campbell, "A novel visible light communication system for enhanced control of autonomous delivery robots in a hospital," 2012 IEEE/SICE International Symposium on System Integration (SII), pp. 510 - 516, 2012.

[8] K. Okuda, T. Yamamoto, T. Nakamura, and W. Uemura, "The Key Providing System for Wireless Lan Using Visible Light Communication," International Journal of Ad hoc, Sensor \& Ubiquitous Computing (IJASUC), vol. 5, pp. 13 - 20, 2014.

[9] K. Okuda, H. Shirai, T. Nakamura, and W. Uemura, "A Novel Keyless Entry System Using Visible Light Communication," International Journal of Ad hoc, Sensor \& Ubiquitous Computing (IJASUC), vol. 5 , pp. $1-8,2014$.

[10] K. Okuda, R. Yoneda, T. Nakamura, and W. Uemura, "A Warning System for Overspeed at the Corner Using Visible Light Based Road-To-Vehicle Communication," International Journal of Ad hoc, Sensor \& Ubiquitous Computing (IJASUC), vol. 6, pp. 1-9, 2015.

[11]W. Uemura and T. Kitazawa, "A Hybrid Modulation Method for Dimming in Visible Light Communication," International Journal of Computer Networks \& Communications (IJCNC), to appear. 2018. 
International Journal of Ad hoc, sensor \& Ubiquitous Computing (IJASUC), Vol.9, No.3/4, August 2018

\section{AUTHORS}

Wataru Uemura was born in 1977, and received B.E, M.E. and D.E. degrees from Osaka City University, in 2000, 2002, and 2005. He is an associate professor of the Department of Electronics and Informatics, Faculty of Engineering Science, Ryukoku University in Shiga, Japan. He is a member of IEEE, RoboCup and others.

Kenta Shimizu was born in 1994 and received Bachelor of Engineering degrees in 2018 from Ryukoku University in Shiga, Japan. He is a master course student in JAIST. He is interested in the Design engineering.
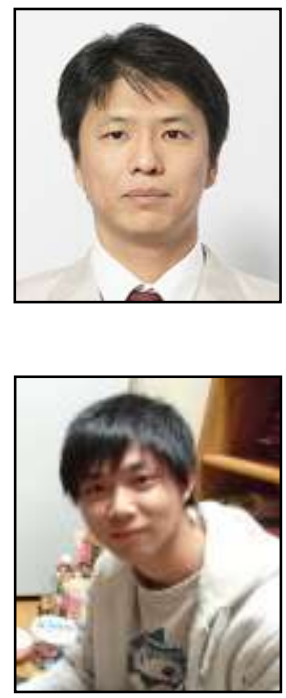